\title{
Intravenous levetiracetam in critically ill children with status epilepticus or acute repetitive seizures
}

\author{
Nicholas S. Abend, MD, Heather M. Monk, PharmD, Daniel J. Licht, MD, and Dennis J. Dlugos, \\ MD \\ Division of Neurology (NSA, DJL, DJD); and Department of Pharmacy Services (HMM), The \\ Children's Hospital of Philadelphia, Philadelphia, PA
}

\begin{abstract}
Objective-Intravenous (IV) levetiracetam (LEV) is approved for use in patients older than 16 years and may be useful in critically ill children, although there is little data available regarding pharmacokinetics. We aim to investigate the safety, an appropriate dosing, and efficacy of IV LEV in critically ill children.
\end{abstract}

Design-We describe a cohort of critically ill children who received IV LEV for status epilepticus, including refractory or nonconvulsive status, or acute repetitive seizures.

Results-There were no acute adverse effects noted. Children had temporary cessation of ongoing refractory status epilepticus, termination of ongoing nonconvulsive status epilepticus, cessation of acute repetitive seizures, or reduction in epileptiform discharges with clinical correlate.

Conclusions-IV LEV was effective in terminating status epilepticus or acute repetitive seizures and well tolerated in critically ill children. Further study is needed to elucidate the role of IV LEV in critically ill children.

\section{Keywords}

levetiracetam; status epilepticus; seizure; pediatric

Intravenous (IV) levetiracetam (LEV) has been approved for use by the U.S. Food and Drug Administration in patients aged 16 years and older (1), but there is little data available regarding pharmacokinetics, tolerability, or efficacy in younger children. Additionally, LEV has not been approved by the Food and Drug Administration for treatment of acute seizures. LEV is a broadspectrum anticonvulsant and can be administered rapidly as a loading dose over 15 minutes, although adult studies suggest infusion times of 5 minutes are well tolerated (2).

Pharmacokinetically, LEV completely avoids hepatic metabolism, which may prove beneficial in complex patients with liver dysfunction or metabolic disorders or those patients at risk for major drug interactions. In comparison with other IV anticonvulsants, LEV has few known adverse effects, including a low risk of sedation, cardiorespiratory depression, or coagulopathy, and thus is potentially useful in critically ill pediatric patients. We present data on a cohort of critically ill children who received IV LEV for status epilepticus or acute repetitive seizures.

\section{Methods}

This is a retrospective case series of critically ill children who received IV LEV for status epilepticus or acute repetitive seizures at The Children's Hospital of Philadelphia between 
August 2006 and January 2008. The study was approved by the Hospital's Institutional Review Board.

Clinical data were obtained from review of the neurology consult team database, hospital records, and electroencephalography (EEG) records. The neurology consult team was involved in the care of all patients as IV LEV was only recently added to the hospital formulary. Patients who were undergoing long-term EEG monitoring had an event marker placed at the time of LEV administration to mark infusion time and judge effect. Patients not already undergoing long-term EEG monitoring were monitored after medication administration to evaluate for nonconvulsive seizures. As part of a clinical protocol, EEG monitoring was continued for at least 24 hours after seizure termination to confirm seizures did not recur. All patients had a serum LEV level drawn within 1 hour of the loading dose administration. Although the serum concentration was not available for 2-3 days and thus could not be used in the initial management, this level was obtained as a baseline to aid in future LEV dosing, specifically in anticipation of patients continued on treatment who would later develop renal dysfunction. If it was thought to be clinically indicated, some patients had additional levels drawn, including troughs, but these were not drawn in a rigorous pharmacokinetic study manner. Children underwent cardiopulmonary monitoring during treatment. Many had routine laboratory testing performed as part of their clinical care, but these were not standardized.

Data gathered included patient demographics, current medical issues (including mental status, respiratory status, cardiovascular status, and reason for intensive care unit [ICU] admission), seizure types and duration, EEG findings, anticonvulsant administration timing and dosing and serum levels, and anticonvulsant effect. Seizures were classified as clinical (observable convulsion) or subclinical (detected by EEG), as focal or generalized, and as repetitive isolated seizures or status epilepticus (seizure longer than 5 minutes) or refractory status epilepticus (seizures not terminated by adequate doses of at least two anticonvulsants) or malignant refractory status epilepticus (seizures recurred after period of pharmacologically induced coma).

\section{Results}

Ten children were identified who received IV LEV for status epilepticus or acute repetitive seizures in the pediatric ICU (Table 1). The median age was 5 years and range was $0.08-14$ years. Loading dose of LEV ranged from 6.5 to $31 \mathrm{mg} / \mathrm{kg}$. Indications for therapy included nonconvulsive status epilepticus refractory to other anticonvulsant medications in three children, nonconvulsive status epilepticus with LEV as the first-line anticonvulsant in two children, acute repetitive seizures in four children (nonconvulsive in two patients, clinical in two patients), and periodic epileptiform discharges with clinical correlate in one child. Eight patients had the seizures detected by long-term EEG monitoring, which allowed evaluation of electrographic effect of LEV administration. The two patients with clinical seizures that did not recur after LEV administration had greater than 24 hours of long-term EEG monitoring performed after LEV administration to ensure nonconvulsive seizures did not persist. Three patients had seizures refractory to treatment with other anticonvulsants before LEV administration whereas in seven patients LEV was administered as the first-line agent. Before LEV administration, three patients were hypotensive, seven were intubated, six were comatose, two were obtunded, and two were lethargic. One patient had renal and hepatic dysfunction before initiation of LEV therapy.

All patients underwent continuous cardiopulmonary monitoring during LEV administration. None of the patients experienced hypotension, hypertension, bradycardia or tachycardia, or respiratory compromise. There were no changes in mental status, although patients with alterations of mental status before administration of LEV remained so acutely. As serum 
laboratory testing was not performed in a standardized manner during clinical care and due to the multiple children with preexisting multiorgan dysfunction, other potential systemic adverse effects could not be addressed.

All children were either undergoing EEG monitoring when seizures were detected or had EEG monitoring initiated after clinical seizures occurred. In patients with seizure termination, EEG monitoring was continued for at least 24 hours after electrographic seizure termination to ensure there was no recurrence. All patients with hypoxic ischemic encephalopathy (two of two) treated with hypothermia had termination of nonconvulsive status epilepticus with LEV as a first anticonvulsant. All patients with malignant refractory nonconvulsive status epilepticus (three of three) had a temporary (12-24 hours) termination of seizures. Three of four patients with acute repetitive seizures had cessation after LEV administration as the first-line anticonvulsant. The fourth had cessation after a second loading dose of LEV administered 1.5 hours after the initial dose. One child with periodic epileptiform discharges with an eye blinking clinical correlate had a greater than $75 \%$ reduction in event frequency with LEV as the firstline anticonvulsant.

Serum levels were drawn within 60 minutes of administration based on previous pharmacokinetic studies (3) and ranged from 8 to $41 \mu \mathrm{g} / \mathrm{mL}$. Although there was a trend that higher loading doses were associated with higher serum levels, the correlation was low $(r=$. 59) (Fig. 1).

One patient (patient 1 in Table) with a history significant for orthotopic heart transplant presented with multisystem organ failure including status epilepticus, acute respiratory failure, acute hepatic insufficiency, coagulopathy, circulatory failure, and renal failure, originating from an acute fungal sepsis and meningoencephalitis. The serum creatinine at initiation of LEV treatment was $3.4 \mathrm{mg} / \mathrm{dL}$, with a serum LEV level of $16 \mu \mathrm{g} / \mathrm{mL} 1$ hour after the initial $200 \mathrm{mg}$ $(6.5 \mathrm{mg} / \mathrm{kg})$ loading dose. The patient was maintained on $200 \mathrm{mg}$ every 12 hours $(13 \mathrm{mg} / \mathrm{kg} /$ day) and LEV levels were drawn approximately every 8 hours. During the course of treatment, the patient experienced a marked decline in renal function, with the serum creatinine increasing from 3.6 to $4.8 \mathrm{mg} / \mathrm{dL}$ over a 16 -hour period. This change in renal function yielded an increase in serum LEV level of 22 to $39 \mu \mathrm{g} / \mathrm{mL}$ (Fig. 2).

\section{Discussion}

LEV, a second-generation antiepileptic drug considered effective in a broad spectrum of seizure types, has bioequivalent oral and IV formulations $(1,2,4)$. Although the IV formulation is only approved for use in adults, the oral formulation has approved indications for children older than 4 years. LEV has been postulated to have a variety of effects, including inhibition of voltage-dependent calcium channel neurotransmitter release, facilitation of GABAergic inhibitory transmission through displacement of negative modulators, reduction of delayed rectifier potassium currents, and binding to synaptic proteins which modulate neurotransmitter release (1).

Adult and pediatric pharmacokinetic studies have demonstrated that LEV exhibits linear pharmacokinetics and children have been shown to have a shorter mean half-life and a more rapid LEV clearance than adults (1,3,5-8). A study of oral LEV solution (single dose of 20 $\mathrm{mg} / \mathrm{kg}$ ) in children aged 2-46 months of age demonstrated these altered kinetics, suggesting infants and small children may require higher initial doses as compared with adults (3). Pharmacokinetic parameters in children older than 6 months were consistent with those in older pediatric patients, whereas children younger than 6 months showed slightly reduced clearance, but still more rapid than adults $(3,6)$. 
LEV clearance is also dependent on renal function. Two-thirds of LEV ingested is excreted in the urine as unchanged drug. Adult studies have shown that half-life is increased in the elderly, primarily due to impaired renal clearance, and in subjects with varying degrees of renal impairment (1). Total body clearance of LEV is decreased by as much as $60 \%$ in patients with creatinine clearances $<30 \mathrm{~mL} / \mathrm{min}$, directly correlating LEV clearance with creatinine clearance (1). Maintenance dosage reduction is recommended in patients with renal impairment. Because of the lack of pharmacokinetic and efficacy data on loading doses of LEV, it is unknown whether renal impairment, often a major consideration in critically ill patients, mandates adjustment in loading dose.

In the single patient with renal dysfunction, a rise in creatinine paralleled an increase in LEV, attributed to accumulation secondary to delayed LEV clearance. It is unknown whether the continued rise in creatinine in this patient was related to LEV administration or disease progression. Although no adverse events were observed in this patient, further study is clearly warranted to determine whether dosage adjustment is necessary for the treatment of status epilepticus in pediatric patients with renal dysfunction. This patient also had acute hepatic insufficiency and coagulopathy; however, these findings do not seem to be worsened with LEV administration.

LEV is $<10 \%$ protein bound and is not extensively metabolized in humans, with complete avoidance of the hepatic cytochrome $\mathrm{P} 450$ system $(1,9)$. Because of its low protein affinity and metabolism, LEV lacks the common drug interactions other antiepileptic drugs possess, including with other antiepileptic drugs in children (10). LEVs favorable pharmacokinetic profile makes it attractive as an agent in the management of critically ill patients with seizures or status epilepticus, as traditional antiepileptic drugs may increase the risk of drug interactions or complicate the liver failure or coagulopathy that can be associated with critical illness.

A large case series in critically ill adults suggested LEV monotherapy was associated with fewer complications compared with other anticonvulsants, primarily phenytoin (11). Case series and reports in adults have suggested that LEV is safe and may be effective in terminating refractory status epilepticus $(12-16)$, and nonconvulsive status epilepticus $(12,17,18)$ without significant systemic side effects $(12,13,17)$.

Although definitive data are lacking, there is increasing evidence that LEV may be safe and effective for treating status epilepticus and acute repetitive seizures in children. A recent case series described ten children who received IV LEV, including two with status epilepticus and two with acute repetitive seizures (19). A 3-week old with cortical dysplasia and 4-month old with nonaccidental head injury, both with status epilepticus refractory to phenytoin and phenobarbital, had a partial decrease in seizure frequency and termination of status epilepticus, respectively. Children with acute repetitive seizures, aged 5 and 16 years, had seizure termination after IV LEV administration (19). Like our study, time to seizure abatement was not included for any patient. These data are important in determining the role of LEV in the treatment of acute seizures and needs to be collected in future studies. Another case described that a 1-day-old term newborn with status epilepticus refractory to phenobarbital, midazolam, and fosphenytoin secondary to bilateral infarcts was terminated within 17 minutes of a $60 \mathrm{mg} /$ $\mathrm{kg}$ oral bolus of LEV (20). Other case reports in children have also demonstrated improvement in nonconvulsive status epilepticus with LEV administration $(21,22)$. Seizure termination has not been shown to have a direct correlation with any specific parameter, including loading dose or serum concentration.

Recent animal studies have demonstrated that LEV treatment during the maintenance phase of status epilepticus diminished or aborted seizures (23), is neuroprotective in culture (24) and 
in animals experiencing status epilepticus (25), and may reduce the epileptogenic effects of status epilepticus (26).

The reference range for LEV serum concentrations has not been established for any seizure type, as studies have been unable to find a causal relationship between serum levels and clinical effects, including this case series. An observational study on the correlation between plasma LEV concentration and clinical response in adults with refractory epilepsy reported a nonsignificant difference in serum levels among responders, nonresponders, and partial responders (27). A curve relating the responder/nonresponder status and plasma LEV concentration suggested a concentration of $11 \mu \mathrm{g} / \mathrm{mL}$ as a threshold of response, with $73 \%$ of responders and $29 \%$ of nonresponders with concentrations above this level. Although not statistically significant, the authors suggested that the likelihood of a response is associated with higher LEV concentrations. There was no significant difference in adverse effects related to serum LEV level. During this study, a linear relationship was observed between loading dose and serum level, just as in previous maintenance dose pharmacokinetic studies (1), but a correlation between level and effect could not be established. Serum levels may not fully explain the clinical effects of LEV as the maximal neurophysiologic change associated with LEV administration occurs many hours after the maximal serum level (28). LEV may induce cellular changes with neurophysiologic implications that persist as the LEV level declines.

In outpatient adult clinical trials evaluating LEV in epilepsy management, the most frequently reported adverse reactions were asthenia, somnolence, dizziness, and coordination difficulties, typically within the first 4 weeks of treatment (1). In pediatric clinical trials, the most common findings were somnolence, accidental injury, hostility, nervousness, and asthenia (1). In the acute setting in which patients already had altered mental status, monitoring or evaluation of these cognitive/behavioral side effects could not be performed. A recently published retrospective chart review evaluated 587 patients younger than 4 years for efficacy and tolerability, reporting $34 \%$ of patients experiencing at least one adverse effect (29). Behavior disturbance/irritability and somnolence were the most commonly reported adverse effects, with difficulty sleeping, increased seizure frequency, dizziness, rash, hypertrichosis, and decreased appetite also noted. Approximately half of those patients experiencing adverse effects required discontinuation of LEV, most frequently due to behavior disturbance and/or irritability. The Food and Drug Administration issued an alert in January 2008, warning healthcare providers that antiepileptic drugs increase the risk of suicidal thoughts and behaviors (30). Reports have also noted dramatic weight loss (31), induced diffuse interstitial lung disease (32), hallucinations (33), encephalopathy induced by combination therapy with valproic acid (34), and seizures induced by LEV use $(35,36)$.

Long-term adverse effects were not assessed in this study. LEV is a commonly used anticonvulsant in the outpatient setting and generally is considered to have a good adverse effect profile in comparison with both older anticonvulsants and some newer anticonvulsants (37). If a child responded to LEV in the ICU, had no adverse effects noted acutely, and had an indication for additional anticonvulsant therapy, continuing LEV would be a reasonable choice (38). However, recent data have demonstrated that while generally superior to older anticonvulsants in terms of adverse events, newer anticonvulsants are also associated with long-term effects (39) including behavior changes $(40,41)$ in humans and endocrine (42) and bone growth effects in animals (43). As further data emerge comparing the newer anticonvulsants for long-term use, decisions regarding appropriate anticonvulsants for acute use in an ICU setting and prolonged use in an outpatient setting may need to be separated.

Additionally, the cause-effect relationship between acute symptomatic seizures and outcome remains unclear. Although acute symptomatic seizures are associated with worse outcome (44), it is unclear whether this reflects an effect of the seizures or of more severe neurologic 
injury that causes the seizures acutely and worse outcome chronically. Studies in adults have demonstrated that acute seizures are associated with markers of worse outcome, such as increased intracranial pressure and elevated lactate (45). Similar studies are needed in children. Studies investigating whether treating acute symptomatic seizures improve long-term outcome are needed. Although it would likely be unethical to randomize to a "no treatment": group, future studies might compare long-term outcome following aggressive vs. less aggressive seizure management because it remains unclear whether the potential side effects from aggressive seizure management are better or worse than brief subclinical seizures.

As we await further prospective data, two common scenarios must be managed in the ICU using existing limited data. First, some children have isolated or repetitive acute symptomatic seizures and require acute administration of an anticonvulsant. Increasing data suggest that LEV may be effective in terminating acute symptomatic seizures and that it may be a reasonable anticonvulsant option in some patients. For instance, benzodiazepine administration may be problematic when observation of mental status is critical and phenytoin administration may be problematic in patients at risk for cardiac rhythm disturbances. Given the limited data available for LEV, a short observation period may be warranted and if seizures persist, then more standard anticonvulsants may be administered. Second, some children will have status epilepticus refractory to standard medications such as benzodiazepines and phenytoin requiring further treatment (46). LEV administration may terminate the status epilepticus. However, given the limited data available, a short 5- to 10-minute trial period is probably appropriate and during this period plans should be made to proceed to coma induction if needed. We clinically use a loading dose of $20 \mathrm{mg} / \mathrm{kg}$ in these situations. If the electroencephalographic seizure burden is reduced with the initial LEV dose and side effects are not noted, then possibly increasing LEV to higher doses is warranted. However, if there is no effect at the lower doses then alternative strategies should be used. Baseline renal function should be evaluated and considered in dosing decisions. Data regarding adverse effects when LEV is used in the ICU are limited, so cardiopulmonary monitoring is likely warranted. Obtaining baseline laboratories may prove beneficial in determining whether future adverse effects may be LEV related because little data exist regarding LEV and systemic dysfunction in critically ill children. If LEV is effective, then we generally provide maintenance doses every 12 hours and obtain a serum level. Although the serum level is not clearly linked to efficacy and it may take several days to obtain a result, this could be useful in determining whether seizure recurrence in the future is related to a reduced LEV level.

This case series, bolstered by previous reports, suggests that LEV may be effective in controlling acute seizures and status epilepticus and has a favorable side effect profile. However, these data consist of case reports and series which are subject to inherent biases. Further prospective study is needed to validate the role of IV LEV in managing seizures and status epilepticus in critically ill children, and would need to confirm the benefit or necessity of a loading dose as well as determine the optimal loading and maintenance dose regimens. Rigorous prospective pharmacokinetic study is needed, with particular focus on dosing issues in patients with renal dysfunction. These studies will require predetermined patient selection criteria, dosing, side effect monitoring, and outcome measures including time to seizure termination after LEV administration. Determining seizure abatement will likely require EEG monitoring to ensure that electrographic seizures do not persist once clinical seizures are terminated.

\section{Acknowledgments}

Dr. Abend received a Young Investigators grant from UCB Pharma. 


\section{References}

1. UCB. Keppra injection (levetiracetam) package insert. 2008.

2. Ramael S, Daoust A, Otoul C, et al. Levetiracetam intravenous infusion: A randomized, placebocontrolled safety and pharmacokinetic study. Epilepsia 2006;47:1128-1135. [PubMed: 16886975]

3. Glauser TA, Mitchell WG, Weinstock A, et al. Pharmacokinetics of levetiracetam in infants and young children with epilepsy. Epilepsia 2007;48:1117-1122. [PubMed: 17442002]

4. Ramael S, De Smedt F, Toublanc N, et al. Single-dose bioavailability of levetiracetam intravenous infusion relative to oral tablets and multiple-dose pharmacokinetics and tolerability of levetiracetam intravenous infusion compared with placebo in healthy subjects. Clin Ther 2006;28:734-744. [PubMed: 16861095]

5. Snoeck E, Jacqmin P, Sargentini-Maier ML, et al. Modeling and simulation of intravenous levetiracetam pharmacokinetic profiles in children to evaluate dose adaptation rules. Epilepsy Res 2007;76:140-147. [PubMed: 17851036]

6. Pellock JM, Glauser TA, Bebin EM, et al. Pharmacokinetic study of levetiracetam in children. Epilepsia 2001;42:1574-1579. [PubMed: 11879369]

7. Fountain NB, Conry JA, Rodriguez-Leyva I, et al. Prospective assessment of levetiracetam pharmacokinetics during dose escalation in 4- to 12-year-old children with partial-onset seizures on concomitant carbamazepine or valproate. Epilepsy Res 2007;74:60-69. [PubMed: 17270398]

8. Toublanc N, Sargentini-Maier ML, Lacroix B, et al. Retrospective population pharmacokinetic analysis of levetiracetam in children and adolescents with epilepsy: Dosing recommendations. Clin Pharmacokinet 2008;47:333-341. [PubMed: 18399714]

9. Sankar, R.; Shields, WD. Levetiracetam. In: Pellock, JM.; Bourgeois, B.; Dodson, WE., editors. Pediatric Epilepsy. Third. New York: Demos; 2008. p. 611-619.

10. Otoul C, De Smedt H, Stockis A. Lack of pharmacokinetic interaction of levetiracetam on carbamazepine, valproic acid, topiramate, and lamotrigine in children with epilepsy. Epilepsia 2007;48:2111-2115. [PubMed: 17651416]

11. Szaflarski JP, Meckler JM, Szaflarski M, et al. Levetiracetam use in critically ill patients. Neurocrit Care 2007;7:140-147. [PubMed: 17607530]

12. Knake S, Gruener J, Hattemer K, et al. Intravenous levetiracetam in the treatment of benzodiazepine refractory status epilepticus. J Neurol Neurosurg Psychiatr 2008;79:588-589. [PubMed: 17898030]

13. Patel NC, Landan IR, Levin J, et al. The use of levetiracetam in refractory status epilepticus. Seizure 2006;15:137-141. [PubMed: 16426869]

14. Rossetti AO, Bromfield EB. Determinants of success in the use of oral levetiracetam in status epilepticus. Epilepsy Behav 2006;8:651-654. [PubMed: 16495155]

15. Abend NS, Florance N, Finkel RS, et al. Intravenous levetiracetam terminates refractory focal status epilepticus. Neurocrit Care 2009;10:83-86. [PubMed: 18181032]

16. Schulze-Bonhage A, Hefft S, Oehl B. Termination of complex partial status epilepticus by intravenous levetiracetam-A case report. J Neurol Neurosurg Psychiatry.

17. Rupprecht S, Franke K, Fitzek S, et al. Levetiracetam as a treatment option in nonconvulsive status epilepticus. Epilepsy Res 2007;73:238-244. [PubMed: 17161587]

18. Farooq MU, Naravetla B, Majid A, et al. IV levetiracetam in the management of nonconvulsive status epilepticus. Neurocrit Care 2007;7:36-39. [PubMed: 17657655]

19. Goraya JS, Khurana DS, Valencia I, et al. Intravenous levetiracetam in children with epilepsy. Pediatr Neurol 2008;38:177-180. [PubMed: 18279751]

20. Shoemaker MT, Rotenberg JS. Levetiracetam for the treatment of neonatal seizures. J Child Neurol 2007;22:95-98. [PubMed: 17608315]

21. Alehan F, Ozcay F, Haberal M. The use of levetiracetam in a child with nonconvulsive status epilepticus. J Child Neurol 2008;23:331-333. [PubMed: 18192652]

22. Trabacca A, Profice P, Costanza MC, et al. Levetiracetam in nonconvulsive status epilepticus in childhood: A case report. J Child Neurol 2007;22:639-641. [PubMed: 17690075] 
23. Mazarati AM, Baldwin R, Klitgaard H, et al. Anticonvulsant effects of levetiracetam and levetiracetam-diazepam combinations in experimental status epilepticus. Epilepsy Res 2004;58:167174. [PubMed: 15120747]

24. Rekling JC. Neuroprotective effects of anticonvulsants in rat hippocampal slice cultures exposed to oxygen/glucose deprivation. Neurosci Lett 2003;335:167-170. [PubMed: 12531459]

25. Gibbs JE, Walker MC, Cock HR. Levetiracetam: Antiepileptic properties and protective effects on mitochondrial dysfunction in experimental status epilepticus. Epilepsia 2006;47:469-478. [PubMed: 16529608]

26. Glien M, Brandt C, Potschka H, et al. Effects of the novel antiepileptic drug levetiracetam on spontaneous recurrent seizures in the rat pilocarpine model of temporal lobe epilepsy. Epilepsia 2002;43:350-357. [PubMed: 11952764]

27. Lancelin F, Franchon E, Kraoul L, et al. Therapeutic drug monitoring of levetiracetam by highperformance liquid chromatography with photodiode array ultraviolet detection: Preliminary observations on correlation between plasma concentration and clinical response in patients with refractory epilepsy. Ther Drug Monit 2007;29:576-583. [PubMed: 17898647]

28. Epstein CM, Girard-Siqueira L, Ehrenberg JA. Prolonged neurophysiologic effects of levetiracetam after oral administration in humans. Epilepsia 2008;49:1169-1173. [PubMed: 18397298]

29. Perry MS, Benatar M. Efficacy and tolerability of levetiracetam in children younger than 4 years: A retrospective review. Epilepsia 2007;48:1123-1127. [PubMed: 17430408]

30. Administration FaD. Information for Healthcare Professionals: Suicidality and Antiepileptic Drugs. 2008

31. Gelisse P, Juntas-Morales R, Genton P, et al. Dramatic weight loss with levetiracetam. Epilepsia 2008;49:308-315. [PubMed: 17825078]

32. Newsome SD, Xue LY, Jennings T, et al. Levetiracetam-induced diffuse interstitial lung disease. J Child Neurol 2007;22:628-630. [PubMed: 17690072]

33. Evcimen H, Kushon D, Jenssen S. Nonepileptic hallucinations in use of levetiracetam. Psychosomatics 2007;48:548-549. [PubMed: 18071105]

34. Bauer J. Encephalopathy induced by levetiracetam added to valproate. Acta Neurol Scand 2008;117:374-376. [PubMed: 18081909]

35. Atefy R, Tettenborn B. Nonconvulsive status epilepticus on treatment with levetiracetam. Epilepsy Behav 2005;6:613-616. [PubMed: 15876554]

36. Chaves J, Sander JW. Seizure aggravation in idiopathic generalized epilepsies. Epilepsia 2005;46 (Suppl 9):133-139. [PubMed: 16302887]

37. Bootsma HP, Ricker L, Diepman L, et al. Long-term effects of levetiracetam and topiramate in clinical practice: A head-to-head comparison. Seizure 2008;17:19-26. [PubMed: 17618131]

38. Glauser TA, Ayala R, Elterman RD, et al. Double-blind placebo-controlled trial of adjunctive levetiracetam in pediatric partial seizures. Neurology 2006;66:1654-1660. [PubMed: 16641323]

39. LaRoche SM. A new look at the second-generation antiepileptic drugs: A decade of experience. Neurologist 2007;13:133-139. [PubMed: 17495757]

40. Zaccara G, Gangemi PF, Cincotta M. Central nervous system adverse effects of new antiepileptic drugs. A meta-analysis of placebo-controlled studies. Seizure 2008;17:405-421. [PubMed: 18262442]

41. Helmstaedter C, Fritz NE, Kockelmann E, et al. Positive and negative psychotropic effects of levetiracetam. Epilepsy Behav 2008;13:535-541. [PubMed: 18583196]

42. Svalheim S, Tauboll E, Surdova K, et al. Long-term levetiracetam treatment affects reproductive endocrine function in female Wistar rats. Seizure 2008;17:203-209. [PubMed: 18155931]

43. Nissen-Meyer LS, Svalheim S, Tauboll E, et al. Levetiracetam, phenytoin, and valproate act differently on rat bone mass, structure, and metabolism. Epilepsia 2007;48:1850-1860. [PubMed: 17634065]

44. Young GB, Jordan KG, Doig GS. An assessment of nonconvulsive seizures in the intensive care unit using continuous EEG monitoring: An investigation of variables associated with mortality. Neurology 1996;47:83-89. [PubMed: 8710130] 
45. Vespa PM, Miller C, McArthur D, et al. Nonconvulsive electrographic seizures after traumatic brain injury result in a delayed, prolonged increase in intracranial pressure and metabolic crisis. Crit Care Med 2007;35:2830-2836. [PubMed: 18074483]

46. Abend NS, Dlugos DJ. Treatment of refractory status epilepticus: Literature review and a proposed protocol. Pediatr Neurol 2008;38:377-390. [PubMed: 18486818] 


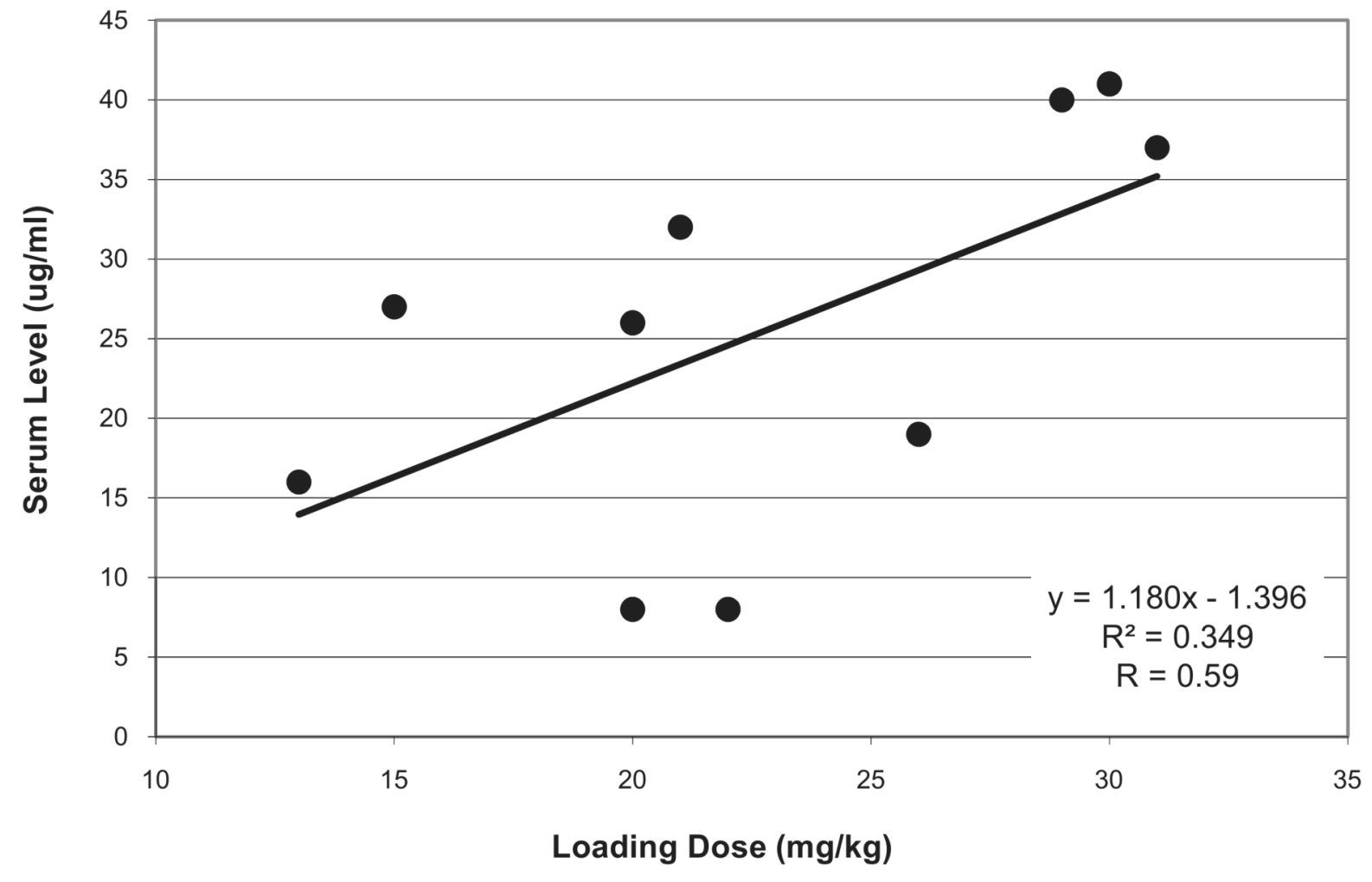

Figure 1.

Levetiracetam loading dose and serum level. 


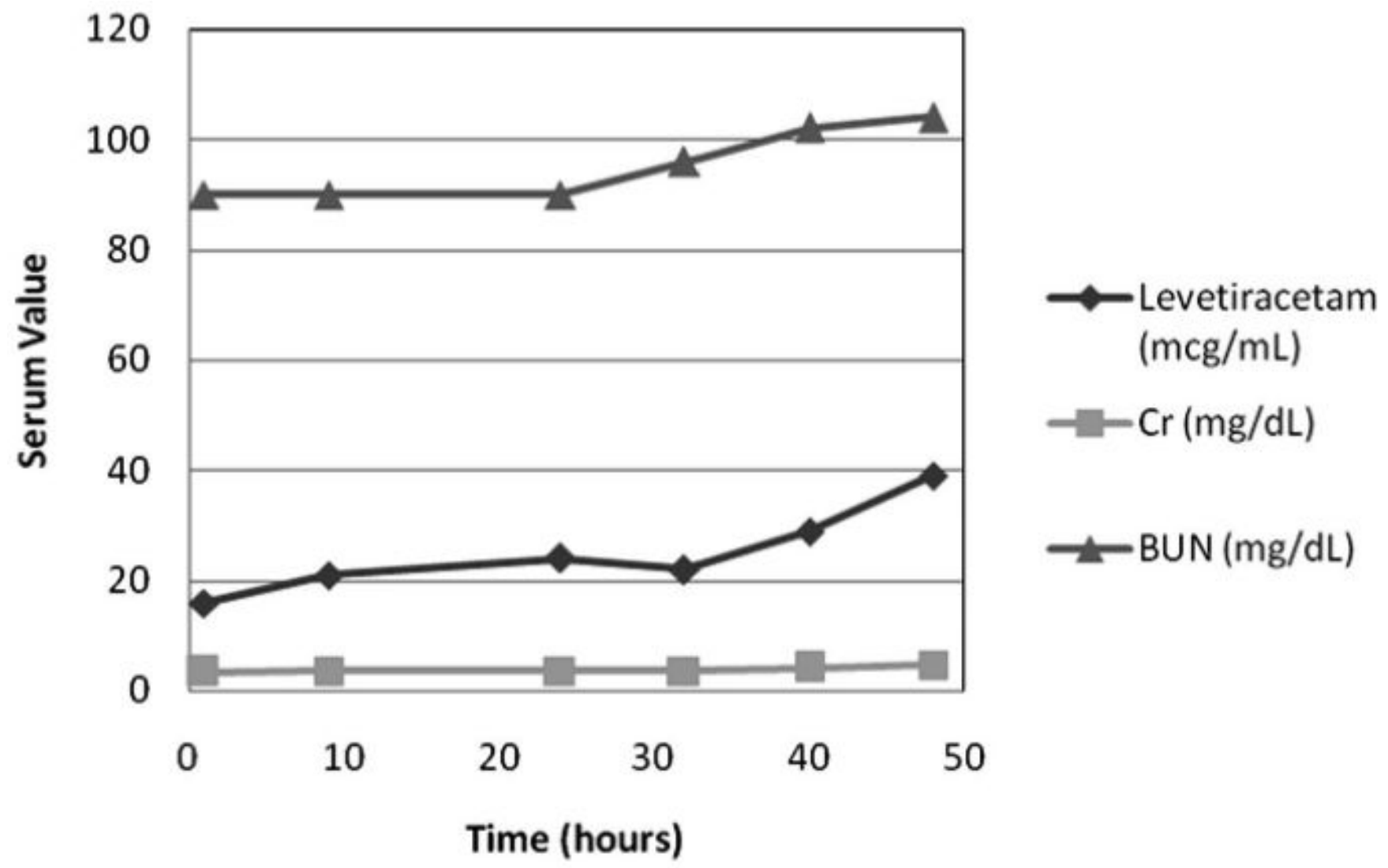

Figure 2.

Serum levetiracetam, creatinine $(\mathrm{Cr})$, and blood urea nitrogen $(B U N)$ levels over time in a patient with renal impairment. 


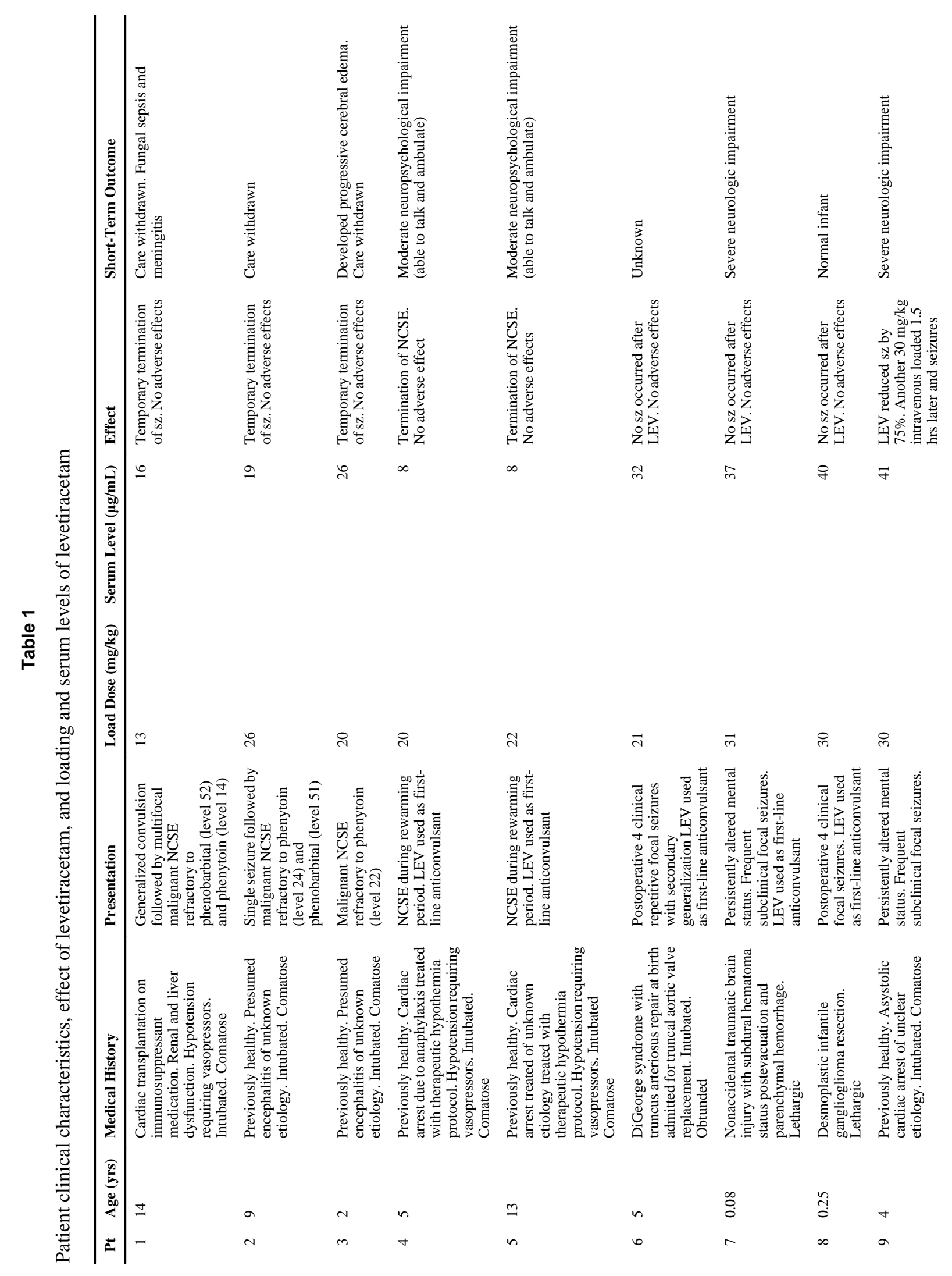

Pediatr Crit Care Med. Author manuscript; available in PMC 2010 September 28. 
Abend et al.

Page 13

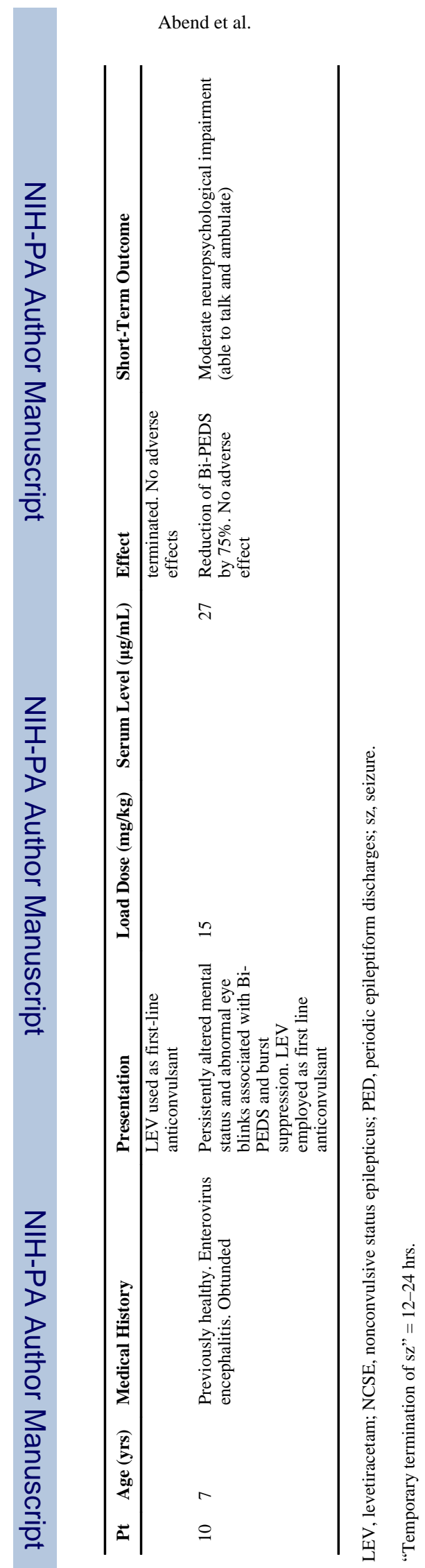

Pediatr Crit Care Med. Author manuscript; available in PMC 2010 September 28. 